\title{
Arbitrary order generalized state space average modeling of switching converters
}

U. Javaid and D. Dujić

This material is posted here with permission of the IEEE. Such permission of the IEEE does not in any way imply IEEE endorsement of any of EPFL's products or services. Internal or personal use of this material is permitted. However, permission to reprint / republish this material for advertising or promotional purposes or for creating new collective works for resale or redistribution must be obtained from the IEEE by writing to pubs-permissions@ieee. org. By choosing to view this document, you agree to all provisions of the copyright laws protecting it. 


\title{
Arbitrary Order Generalized State Space Average Modeling of Switching Converters
}

\author{
Uzair Javaid and Dražen Dujić \\ Power Electronics Laboratory - PEL \\ École Polytechnique Fédérale de Lausanne - EPFL \\ Station 11, CH-1015 Lausanne \\ uzair.javaid@epfl.ch,drazen.dujic@epfl.ch
}

\begin{abstract}
Generalized State Space Average Modeling (GSSAM), of switching converters, offers an opportunity to improve fidelity of a model by inclusion of different harmonic components. Yet, the inclusion of each frequency component contributes to an increase in the number of the state variables and matrices describing the system. In this paper, automated arbitrary order generation of the GSSAM models for switched DC-DC converters is described, which uses State Space Average Model (SSAM) as starting data set. The buck, boost and buck-boost converters are used as examples and comparison between SSAM, GSSAM of different order and PLECS switched model has been carried out to demonstrate improvements in fidelity of models. Finally, closed loop control with arbitrary order GSSAM is shown using Buck converter as an example.
\end{abstract}

\section{INTRODUCTION}

Switched converters have periodic variations in their response, caused by the continuous change in state of the switches governing the conversion process. This change of state entails two sets of differential equations describing the system, one during the 'on' state and the other during the 'off' state of the switch [1]. To overcome this, state space averaging modeling (SSAM) has been proposed [2], but it has a limited applicability to power converters. This limitation arises from the consideration of different approximations i.e. small ripple approximation and linear ripple approximation. Small ripple approximation assumes that $\mathrm{dc}$ term is the dominant term in the circuit waveform, while linear ripple approximation assumes that the response is a linear function of time [3].

A more general averaging approach for switched converters has been proposed in [3]. This general averaging approach is assumed to be applicable to arbitrary waveforms and Fourier representation of a time dependent waveform forms the basis of this approach. This representation yields a linear time invariant system of differential equations and the state variables are the coefficients of developed Fourier series. Therefore, possibilities to include higher number of harmonics increase the accuracy of the averaged model [1]. This approach is known as generalized state space average modeling (GSSAM), and it provides better insight into dynamic behavior of a switched converter than the state space average model (SSAM). Since GSSAM uses frequency-selective averaging, inclusion of oscillations resulting from the switching actions, is possible.
GSSAM has been addressed in [4], [5]. In [4] authors have shown how GSSAM models can be used in the open-loop and the closed-loop configuration for producing accurate variable estimates, while [5] focuses on closed loop behavior and methods to develop condensed models for switched converters. In [1] GSSAM designs for different converters like buck, boost, buck-boost and Cuk, have been presented. It has been shown how changing different parameters (duty ratio, no. of harmonics) of the generalized model influences the overall performance of the model. In [6], this modeling technique has been applied to a system with multiple dc/dc converters, targeting on-board automotive application. GSSAM modeling technique has been further extended to $\mathrm{dc} / \mathrm{ac}$ and $\mathrm{ac} / \mathrm{dc}$ converters [7]-[10] considering both two-level and multilevel inverters and including some of the internal balancing problems associated with these topologies.

In majority of the available literature, presented models do not consider more than two harmonics during the modeling, and mainly restricted to a dc and switching frequency component. This could be partly due to tedious mathematical development needed to derive parameterized analytical expressions for all elements of the state space matrix. Any addition of extra harmonic components for a state variable of interest increases the size of a system due to the use of complex Fourier coefficients. In this paper, we have explored and implemented a numerical algorithm to generate arbitrary order GSSAM models taking input from user to specify number of harmonics. As discussed in the paper, starting point is the easily derivable SSAM model (zero order), which is then expanded to a desired higher order GSSAM model. The exact level of harmonic components can be specified independently for each state variable and at the same time it can be set to be of different order for different state variables.

This paper is organized as follows: Section 2 briefly describes mathematical formulation behind the GSSAM, which serves as foundation for the next steps. Developed numerical algorithm for arbitrary order GSSAM and its MATLAB based implementation is discussed in Section 3. Various simulation results are provided in Section 4, including the open- and closed-loop operation and comparisons with switched models. Summary and concluding remarks are provided in Section 5. 


\section{Generalized State-SPACE AVERAging}

To capture different harmonic components, frequencyselective averaging is based on expressing any electrical signal in terms of its Fourier series. Eq. (1) shows a function $f(x)$ represented in its Fourier series form:

$$
f(x)=\frac{a_{0}}{2}+\sum_{n=1}^{\infty}\left(a_{n} \cos n x+b_{n} \sin n x\right)
$$

where $a_{0}$ is the dc term of the function while $a_{n}$ and $b_{n}$ are coefficients of corresponding frequency components of different order. Considering Euler's formulas:

$$
\cos n x=\frac{e^{j n x}+e^{-j n x}}{2}, \quad \sin n x=\frac{e^{j n x}-e^{-j n x}}{2 j}
$$

complex Fourier representation of the function $f(x)$ is easily obtained as:

$$
\begin{aligned}
f(x) & =\frac{a_{0}}{2}+\sum_{n=1}^{\infty}\left(a_{n} \frac{e^{j n x}+e^{-j n x}}{2}+b_{n} \frac{e^{j n x}-e^{-j n x}}{2 j}\right) \\
& =\frac{a_{0}}{2}+\sum_{n=1}^{\infty} e^{j n x} \frac{a_{n}-j b_{n}}{2}+\sum_{n=1}^{\infty} e^{-j n x} \frac{a_{n}+j b_{n}}{2} \\
f(x) & =\sum_{n=-\infty}^{\infty} c_{n} e^{j n x}
\end{aligned}
$$

Now consider a signal $x(\tau)$ on the interval $\tau \in[t-T, t]$. Its Fourier series representation is:

$$
x(\tau)=\sum_{k=-\infty}^{\infty}\langle x\rangle_{k}(t) e^{j k \omega_{s} t}
$$

where $\omega_{s}=2 \pi / T$ and $T$ is the switching interval or the time window over which the average is calculated and $\langle x\rangle_{k}(t)$ are the complex Fourier coefficients of the signal, which are given by Eq. (5):

$$
\langle x\rangle_{k}(t)=\frac{1}{T} \int_{t-T}^{t} x(\tau) e^{-j k \omega_{s} \tau} \mathrm{d} \tau
$$

with $k$ being the $k$ th coefficient of the signal. Further, $x(\tau)$ reconstructed from its Fourier coefficients is expressed in Eq. (6):

$$
\begin{aligned}
x(\tau)=\langle x\rangle_{0}+\sum_{k=1}^{\infty}\left[\operatorname{Re}\left\{\langle x\rangle_{k}\right\}\right. & \cos \left(k \omega_{s} \tau\right) \\
& \left.-\operatorname{Im}\left\{\langle x\rangle_{k}\right\} \sin \left(k \omega_{s} \tau\right)\right]
\end{aligned}
$$

where $\operatorname{Re}\left\{\langle x\rangle_{k}\right\}$ and $\operatorname{Im}\left\{\langle x\rangle_{k}\right\}$ are the real and imaginary part of $\langle x\rangle_{k}$. The notation $t$ is omitted to simplify the representation. As a reminder and as explained in [5], the SSAM model is obtained by considering an average over one cycle of the signal:

$$
\langle x\rangle_{0}(t)=\frac{1}{T} \int_{t-T}^{t} x(\tau) \mathrm{d} \tau
$$

From the point of view of the GSSAM, the SSAM considers only the zero-order component of the signal, which is the dc term of Fourier series as given in Eq. (6). Nevertheless, for many converters these SSAM models are well known or are easy to derive, considering only few state variables and/or control inputs.

Two important properties of the frequency-selective averaging play a pivotal role in the development of the GSSAM. These are:

1) Differentiation of the index- $k$ average with respect to time

2) Computation of the index- $k$ average of the product of two signals

Details related to the implementation of these properties are provided next as they are highly relevant for the arbitrary order GSSAM model development.

\section{A. Differentiation with respect to time}

This property helps in computing the derivative of a function represented in its complex Fourier form. To apply this property on Eq. (5), a mathematical theorem describing differentiation over integral is used [11]. This theorem states that:

$$
\frac{\mathrm{d}}{\mathrm{d} t} \int_{a}^{b} f(x, t) d x=\int_{a}^{b} \frac{\partial}{\partial t} f(x, t) d x
$$

Eq. (8) is valid at $t=t_{0}$, in the sense that both sides exist and are equal, provided the following two conditions hold:

1) $f(x, t)$ and $\frac{\partial}{\partial t} f(x, t)$, are continuous functions of two variables $x$ when it is in the range of integration and $t$ is in some interval around.

2) there are upper bounds $|f(x, t)| \leq A(x)$ and $\left|\frac{\partial}{\partial t} f(x, t)\right| \leq B(x)$, both being independent of $t$, such that $\int_{a}^{b} A(x) d x$ and $\int_{a}^{b} B(x) d x$ exist.

Applying Eq. (8) on Eq. (5) results in:

$$
\frac{\mathrm{d}}{\mathrm{d} \tau}\langle x\rangle_{k}=\frac{1}{T} \int_{t-T}^{t} \frac{\partial}{\partial \tau}\left(x(\tau) e^{-j k \omega_{s} \tau}\right) \mathrm{d} \tau
$$

and after using 'product rule' one has:

$$
\begin{aligned}
\frac{\mathrm{d}}{\mathrm{d} \tau}\langle x\rangle_{k} & =\frac{1}{T} \int_{t-T}^{t}\left[x(\tau) \frac{\partial}{\partial \tau}\left(e^{-j k \omega_{s} \tau}\right)+e^{-j k \omega_{s} \tau} \frac{\partial}{\partial \tau} x(\tau)\right] \mathrm{d} \tau \\
& =\left\langle\frac{\mathrm{d}}{\mathrm{d} \tau} x\right\rangle_{k}-j k \omega_{s}\langle x\rangle_{k}
\end{aligned}
$$

Result presented in Eq. (10) will help to determine frequency-selective average of the state variables.

\section{B. Computation of average of the product}

The computation of the average of the product of state variables/input signals or any combination of these two can be evaluated using discrete convolution of two signals $(f(t)$ and $g(t)$ ), which is given in Eq. (11).

$$
\langle f g\rangle_{k}=\sum_{i=-\infty}^{\infty}\langle f\rangle_{k-i}\langle g\rangle_{i}
$$


Proof of Eq. (11) is easy to determine. Let us consider arbitrary electrical signals $f(t)$ and $g(t)$ that can be represented in terms of their kth harmonics.

$$
\begin{aligned}
f(t) \approx & \langle f\rangle_{-k} e^{-j k \omega_{s} t}+\cdots+\langle f\rangle_{-1} e^{-j \omega_{s} t}+\langle f\rangle_{0} \\
& +\langle f\rangle_{1} e^{j \omega_{s} t}+\cdots+\langle f\rangle_{k} e^{j k \omega_{s} t} \\
g(t) \approx & \langle g\rangle_{-k} e^{-j k \omega_{s} t}+\cdots+\langle g\rangle_{-1} e^{-j \omega_{s} t}+\langle g\rangle_{0} \\
& +\langle g\rangle_{1} e^{j \omega_{s} t}+\cdots+\langle g\rangle_{k} e^{j k \omega_{s} t}
\end{aligned}
$$

Substituting Eq. (12) in Eq. (11) will yield average product for different values of $k$. For illustration, lets consider that $f(t)$ and $g(t)$ are approximated by the sum of their index0 and index- 1 terms i.e. the dc component and fundamental frequency component, while neglecting all higher frequency terms. Case for $k=0,1,-1$ yields:

$$
\begin{aligned}
\langle f g\rangle_{0} & =\langle f\rangle_{0}\langle g\rangle_{0}+\langle f\rangle_{-1}\langle g\rangle_{1}+\langle f\rangle_{1}\langle g\rangle_{-1} \\
\langle f g\rangle_{1} & =\langle f\rangle_{0}\langle g\rangle_{1}+\langle f\rangle_{1}\langle g\rangle_{0} \\
\langle f g\rangle_{-1} & =\langle f\rangle_{0}\langle g\rangle_{-1}+\langle f\rangle_{-1}\langle g\rangle_{0}
\end{aligned}
$$

From Eq. (5) it can be concluded that the terms $\langle\cdot\rangle_{ \pm 1}$ are complex terms and can be written in real and imaginary form:

$$
\begin{aligned}
\langle f\rangle_{k} & =\langle f\rangle_{k}^{\Re}+j\langle f\rangle_{k}^{\Im}=\langle f\rangle_{-k}^{*}=\left[\langle f\rangle_{-k}^{\Re}+j\langle f\rangle_{-k}^{\Im}\right]_{(14 \mathrm{a})}^{*} \\
\langle g\rangle_{k} & =\langle g\rangle_{k}^{\Re}+j\langle g\rangle_{k}^{\Im}=\langle g\rangle_{-k}^{*}=\left[\langle g\rangle_{-k}^{\Re}+j\langle g\rangle_{-k}^{\Im}\right]^{*}(14 \mathrm{~b})
\end{aligned}
$$

Inserting Eq. (13) in Eq. (14) gives expressions for the average of product of two signals as a function of average of real and imaginary parts of each signal:

$$
\begin{aligned}
\langle f g\rangle_{0}= & \langle f\rangle_{0}\langle g\rangle_{0}+2\left[\langle f\rangle_{1}^{\Re}\langle g\rangle_{1}^{\Re}+\langle f\rangle_{1}^{\Im}\langle g\rangle_{1}^{\Im}+\ldots\right. \\
& \left.+\langle f\rangle_{k}^{\Re}\langle g\rangle_{k}^{\Re}+\langle f\rangle_{k}^{\Im}\langle g\rangle_{k}^{\Im}\right] \\
\langle f g\rangle_{k}^{\Re}= & \Re\left\{\sum_{i=-\infty}^{\infty}\langle f\rangle_{k-i}\langle g\rangle_{i}\right\} \\
\langle f g\rangle_{k}^{\Im}= & \Im\left\{\sum_{i=-\infty}^{\infty}\langle f\rangle_{k-i}\langle g\rangle_{i}\right\}
\end{aligned}
$$

\section{Input control signal}

To control the converter, there will be at least one control signal $u(t)$ which can be expressed as:

$$
u(t)= \begin{cases}1, & 0<t<\mathrm{d} t \\ 0, & \mathrm{~d} t<t<T\end{cases}
$$

with $d$ being the duty cycle. To find $\langle u\rangle_{0}$ and $\langle u\rangle_{k}$, one can apply Eq. (5) on Eq. (16). As $\langle u\rangle_{0}$ is the dc-component of the signal, it can be calculated as:

$$
\langle u\rangle_{0}=\frac{1}{T} \int_{0}^{T} u(t) \mathrm{d} t=d
$$

The calculation of $\langle u\rangle_{k}$ yields:

$$
\begin{aligned}
\langle u\rangle_{k} & =\frac{1}{T} \int_{0}^{T} u(t) e^{-j k \omega_{s} t} \mathrm{~d} t=\frac{j}{2 \pi k}\left(e^{-j 2 \pi k d}-1\right) \\
& =\frac{\sin 2 \pi k d+j(\cos 2 \pi k d-1)}{2 \pi k}
\end{aligned}
$$

\section{ARbitrary ORder GSSAM DEVElOPMENT}

Usually, harmonics higher than '1' have not been implemented for averaged models. To generate models that include higher harmonics, a large amount of mathematical manipulations are required. To circumvent this an algorithm has been developed, which can generate a model with large number of harmonics using mathematical considerations from section 2 . This algorithm uses symbolic toolbox of MATLAB and user can set desired number of harmonics to be included in the model beyond the starting SSAM model. Symbolic variables are generated accordingly for each differential equation defining the state of the system. The number of component variables generated for each state variable is given by:

$$
h_{m}=2 k_{m}+1
$$

where $k=0,1,2,3, \ldots$ is the harmonic order and $m=$ $1,2, \ldots, q$ are different state variable. Using this, variables for the dc term (0th order harmonic) and real and imaginary part of the harmonic components are all generated. Simplified steps for automatic GSSAM generation are:

- Define symbolic variables using Eq. (19). The total number of variables generated depends on the number of state variables $s_{m}$ of the system i.e. $h_{\text {tot }}=h_{1}+h_{2}+\cdots+h_{q}$, where $h_{1}=2 k_{1}+1, h_{2}=2 k_{2}+1, \ldots, h_{q}=2 k_{q}+1$ and $k_{1}, k_{2}, \ldots, k_{q}$ are the harmonics of each state variable. Therefore, the number of differential equations to be solved is $h_{t o t}$.

- Calculate Fourier series of switching signal (given here by Eq. (17) and Eq. (18)) and input voltage by using Eq. (5) and Eq. (7).

- Compute convolution terms using Eq. (15) and input these terms into system differential equations.

- Separate input signals from the differential equations. The complex equations should be further separated into real and imaginary parts.

Following these steps reduces the system complexity during mathematical calculations. To avoid calculation of positive and negative component of harmonics, we only calculate the real and imaginary part of harmonics and by using Eq. (14) can determine either of them. A generalized form of the differential equations in characteristic state space matrix form is given as:

$$
\begin{aligned}
& \dot{x}=A x+B u \\
& y=C x
\end{aligned}
$$

Developed form, which provides insight into all state space variable and their corresponding harmonic components is given in Eq. (21), where $\omega=2 \pi f, v_{u}$ is the number of variables for input $h_{u}=2 k_{u}+1$ and $k_{u}$ is the harmonic order considered for input. Eq. (20) shows how actual state space variable $s_{1}$ is assembled out of its harmonic parts $\left[\begin{array}{llll}x_{1} & x_{2} & \ldots & x_{h_{1}}\end{array}\right]$. In a similar fashion, other $q-1$ state space variables can be recovered.

Models for basic buck, boost and buck-boost converters are given below. Presented examples consider only 0th and 1st 


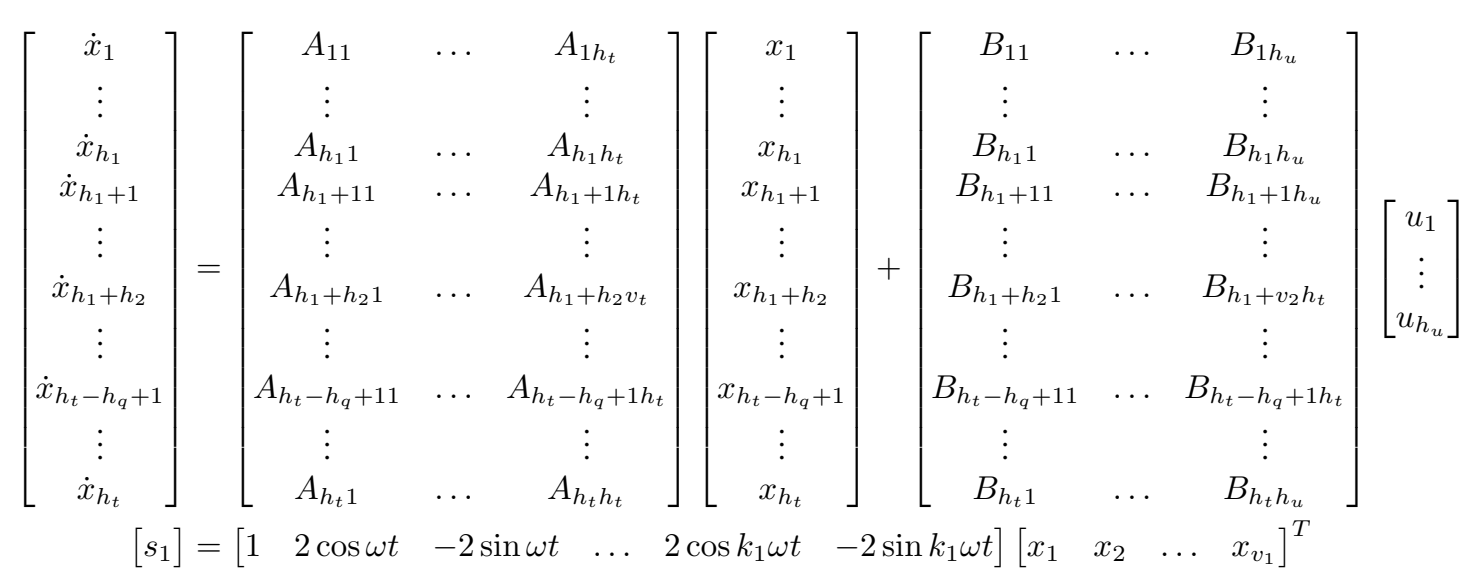

harmonic component resulting in six state space variables to be computed, as often reported in literature. Further addition of the 2nd harmonic would increase the size of the system to ten state space variables. Numerical arbitrary order GSSAM model generation simplifies and speed-up these developments as described in subsequent sections.

\section{A. Buck converter}

Basic buck converter as shown in the Fig. 1. The following equations describe the continuous conduction mode (CCM) of the converter when $u(t)$, as given in Eq. (16) is applied:

$$
\begin{aligned}
\frac{\mathrm{d}}{\mathrm{d} t} i_{L} & =\frac{1}{L}\left(v_{i n} u(t)-v_{c}\right) \\
\frac{\mathrm{d}}{\mathrm{d} t} v_{C} & =\frac{1}{C}\left(i_{L}-\frac{v_{C}}{R}\right)
\end{aligned}
$$

As a starting point conventional state space model (SSAM) for this converter is given as:

$$
\left[\begin{array}{l}
\dot{x}_{1} \\
\dot{x}_{2}
\end{array}\right]=\left[\begin{array}{cc}
0 & -\frac{1}{L} \\
\frac{1}{C} & -\frac{1}{R C}
\end{array}\right]\left[\begin{array}{l}
x_{1} \\
x_{2}
\end{array}\right]+\left[\begin{array}{c}
\frac{d}{L} \\
0
\end{array}\right] v_{i n}
$$

$i_{L}$ and $v_{C}$ can be represented in terms of their Fourier coefficients similar to Eq. (12) and with Eq. (15) we can further express them in terms of real and imaginary components of the harmonics. To develop the GSSM model for buck converter, we apply Eq. (10) on Eq. (22). This yields:

$$
\begin{aligned}
\frac{\mathrm{d}}{\mathrm{d} t}\left\langle i_{L}\right\rangle_{k} & =\frac{1}{L}\left(\left\langle v_{i n} u\right\rangle_{k}-\left\langle v_{C}\right\rangle_{k}\right)-j k \omega_{s}\left\langle i_{L}\right\rangle_{k} \\
\frac{\mathrm{d}}{\mathrm{d} t}\left\langle v_{C}\right\rangle_{k} & =\frac{1}{C}\left(\left\langle i_{L}\right\rangle_{k}-\frac{\left\langle v_{C}\right\rangle_{k}}{R}\right)-j k \omega_{s}\left\langle v_{C}\right\rangle_{k}
\end{aligned}
$$

For simplicity we consider 0 and 1 for $k$ and $v_{i n}$ to be pure DC, resulting in complete model as shown in Eq. (26). On the other hand, we can represent $i_{L}(t)$ and $v_{C}(t)$ as:

$$
\begin{aligned}
i_{L}(t) & =x_{1}+2 x_{2} \cos \omega t-2 x_{3} \sin \omega t \\
v_{C}(t) & =x_{4}+2 x_{5} \cos \omega t-2 x_{6} \sin \omega t
\end{aligned}
$$

\section{B. Boost converter}

Consider the boost converter as shown in the Fig. 2. The $\mathrm{CCM}$ operation of the boost converter is described with:

$$
\begin{aligned}
\frac{\mathrm{d}}{\mathrm{d} t} i_{L} & =\frac{1}{L}\left(v_{i n}-(1-u(t)) v_{C}\right) \\
\frac{\mathrm{d}}{\mathrm{d} t} v_{C} & =\frac{1}{C}\left((1-u(t)) i_{L}-\frac{v_{C}}{R}\right)
\end{aligned}
$$

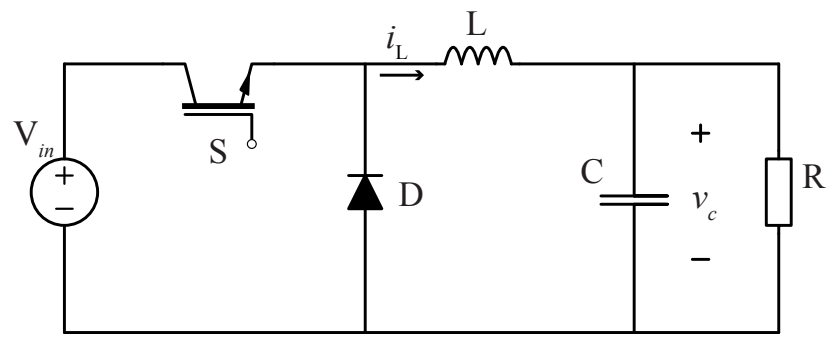

Fig. 1. Buck converter circuit

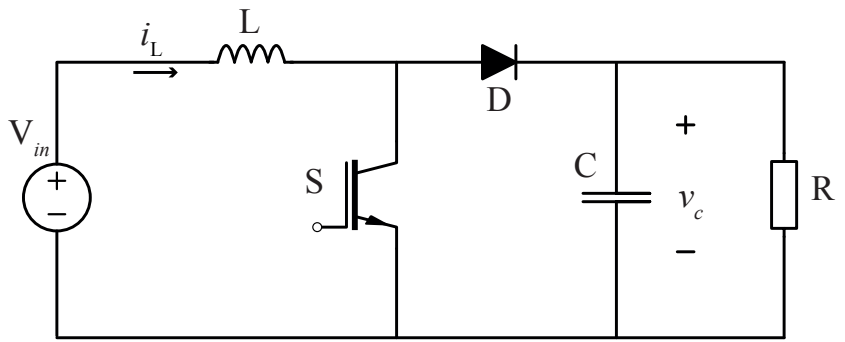

Fig. 2. Boost converter circuit 
This result in a conventional state space model for the boost converter:

$$
\left[\begin{array}{l}
\dot{x}_{1} \\
\dot{x}_{2}
\end{array}\right]=\left[\begin{array}{cc}
0 & -\frac{1-d}{L} \\
\frac{1-d}{C} & -\frac{1}{R C}
\end{array}\right]\left[\begin{array}{l}
x_{1} \\
x_{2}
\end{array}\right]+\left[\begin{array}{l}
\frac{1}{L} \\
0
\end{array}\right] v_{i n}
$$

Following the same procedure as described earlier and considering 0 and 1 for $k$ and $v_{i n}$ to be pure DC, the model is given in Eq. (29). $i_{L}$ and $v_{C}$, in this case, are similar to Eq. (25).

\section{Buck-Boost converter}

As the final example, the buck-boost converter as shown in the Fig. 3, while the relevant equations describing the CCM mode of operation are:

$$
\begin{aligned}
\frac{\mathrm{d}}{\mathrm{d} t} i_{L} & =\frac{1}{L}\left(v_{i n} u(t)-(1-u(t)) v_{C}\right) \\
\frac{\mathrm{d}}{\mathrm{d} t} v_{C} & =\frac{1}{C}\left((1-u(t)) i_{L}-\frac{v_{C}}{R}\right)
\end{aligned}
$$

The conventional state space model for Buck-Boost converter is given in Eq. (31).

$$
\left[\begin{array}{l}
\dot{x}_{1} \\
\dot{x}_{2}
\end{array}\right]=\left[\begin{array}{cc}
0 & \frac{1-d}{L} \\
\frac{1-d}{C} & -\frac{1}{R C}
\end{array}\right]\left[\begin{array}{l}
x_{1} \\
x_{2}
\end{array}\right]+\left[\begin{array}{l}
\frac{d}{L} \\
0
\end{array}\right] v_{i n}
$$

Following the same procedure, as described earlier, and considering 0 and 1 for $k$ and $v_{i n}$ to be pure DC, the model is given in Eq. (32). $i_{L}$ and $v_{C}$, in this case, are similar to Eq. (25).

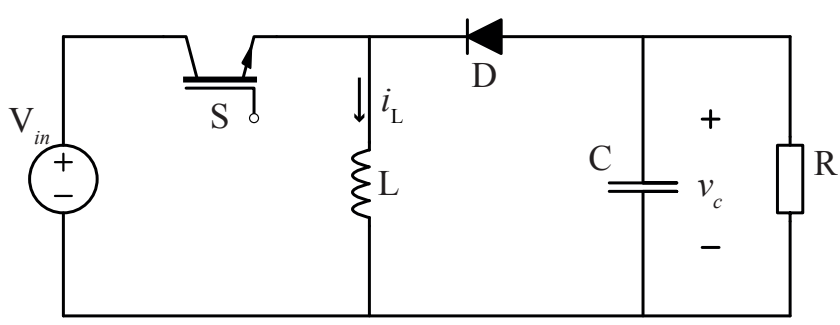

Fig. 3. Buck-Boost converter circuit

\section{Simulation AND RESUlts}

\section{A. Open-loop simulation}

At first, open loop simulations were carried out for arbitrary order GSSAMs for buck, boost and buck-boost converters. Higher order models have been generated directly using symbolic toolbox of MATLAB, as described in Section III. The mathematical models for the case $k=0$ are given in Eqs. (23), (28) and (31) and similarly for $k=1$ in Eqs. (26), (29) and (32) for buck, boost and buck-boost converters, respectively. In all simulations, all state variables are considered to have the same order of harmonics i.e. $k_{1}=k_{2}=\cdots=k_{q}=k$. Cases with different values of $k$ i.e. $k=0,1,10,25$ are considered for different duty cycles of the switching signal $(d=0.25$ and $d=0.75)$ and compared with switched models implemented in the PLECS. Switching frequency used in simulations is set as $f_{s}=100 \mathrm{kHz}$, while other relevant electrical parameters for all cases are: $V_{i n}=20 \mathrm{~V}, R=10 \Omega, C=10 \mu \mathrm{F}$ and $L=1 \mathrm{mH}$.

Fig. 4, Fig. 5 and Fig. 6 show inductor current and capacitor voltage (state variables) for the buck, boost and buck-boost converters respectively. Open-loop responses to a step change of duty cycle $d$ for GSSAM models of different order are compared directly with equivalent switched model in PLECS. From the zoom-in insets, shown in all plots, it can be seen that inclusion of more high order harmonics of the GSSAM models increases fidelity and captures ripple components of inductor current or capacitor voltage closer to the results obtained from the PLECS models. Compared to SSAM model $(k=0)$ where only average values of inductor current or capacitor voltage are present, the GSSAM model with $k=1$ adds ripple component as sinusoidal components at the switching frequency, while further increase of $k$ results in a closer piece-wise linear ripple representation. This effect can be equally observed for all three converter circuits considered.

In all open-loop simulations, the actual order of higher harmonics $k$ has been set the same for both state variables. Implemented algorithm, however, allows for the harmonic order to be independently defined for each state variable, depending on the user settings. In this way different state variables can be observed with different level of details, and the actual size and complexity of the model can be controlled.

$$
\left[\begin{array}{c}
\dot{x}_{1} \\
\dot{x}_{2} \\
\dot{x}_{3} \\
\dot{x}_{4} \\
\dot{x}_{5} \\
\dot{x}_{6}
\end{array}\right]=\left[\begin{array}{cccccc}
0 & 0 & 0 & -\frac{1}{L} & 0 & 0 \\
0 & 0 & \omega & 0 & -\frac{1}{L} & 0 \\
0 & -\omega & 0 & 0 & 0 & -\frac{1}{L} \\
\frac{1}{C} & 0 & 0 & -\frac{1}{R C} & 0 & 0 \\
0 & \frac{1}{C} & 0 & 0 & -\frac{1}{R C} & \omega \\
0 & 0 & \frac{1}{C} & 0 & -\omega & -\frac{1}{R C}
\end{array}\right]\left[\begin{array}{c}
x_{1} \\
x_{2} \\
x_{3} \\
x_{4} \\
x_{5} \\
x_{6}
\end{array}\right]+\left[\begin{array}{c}
\frac{d}{L} \\
0 \\
0 \\
0 \\
0 \\
0
\end{array}\right]\left\langle v_{i n}\right\rangle_{0}
$$




$$
\begin{aligned}
& {\left[\begin{array}{l}
\dot{x}_{1} \\
\dot{x}_{2} \\
\dot{x}_{3} \\
\dot{x}_{4} \\
\dot{x}_{5} \\
\dot{x}_{6}
\end{array}\right]=\left[\begin{array}{cccccc}
0 & 0 & 0 & -\frac{1-d}{L} & \frac{\sin 2 \pi d}{\pi L} & \frac{\cos \pi d-1}{\pi L} \\
0 & 0 & \omega & \frac{\sin 2 \pi d}{\pi L} & -\frac{1-d}{L} & 0 \\
0 & -\omega & 0 & \frac{\cos \pi d-1}{\pi L} & 0 & -\frac{1-d}{L} \\
\frac{1-d}{C} & -\frac{\sin 2 \pi d}{\pi C} & -\frac{\cos \pi d-1}{\pi C} & -\frac{1}{R C} & 0 & 0 \\
-\frac{\sin 2 \pi d}{\pi C} & \frac{1-d}{C} & 0 & 0 & -\frac{1}{R C} & \omega \\
-\frac{\cos \pi d-1}{\pi C} & 0 & \frac{1-d}{C} & 0 & -\omega & -\frac{1}{R C}
\end{array}\right]\left[\begin{array}{c}
x_{1} \\
x_{2} \\
x_{3} \\
x_{4} \\
x_{5} \\
x_{6}
\end{array}\right]+\left[\begin{array}{c}
\frac{1}{L} \\
0 \\
0 \\
0 \\
0 \\
0
\end{array}\right]\left\langle v_{i n}\right\rangle_{0}} \\
& {\left[\begin{array}{l}
\dot{x}_{1} \\
\dot{x}_{2} \\
\dot{x}_{3} \\
\dot{x}_{4} \\
\dot{x}_{5} \\
\dot{x}_{6}
\end{array}\right]=\left[\begin{array}{cccccc}
0 & 0 & 0 & \frac{1-d}{L} & -\frac{\sin 2 \pi d}{\pi L} & -\frac{\cos \pi d-1}{\pi L} \\
0 & 0 & \omega & -\frac{\sin 2 \pi d}{\pi L} & \frac{1-d}{L} & 0 \\
0 & -\omega & 0 & -\frac{\cos \pi d-1}{\pi L} & 0 & \frac{1-d}{L} \\
-\frac{1-d}{C} & \frac{\sin 2 \pi d}{\pi C} & \frac{\cos \pi d-1}{\pi C} & -\frac{1}{R C} & 0 & 0 \\
\frac{\sin 2 \pi d}{\pi C} & -\frac{1-d}{C} & 0 & 0 & -\frac{1}{R C} & \omega \\
\frac{\cos \pi d-1}{\pi C} & 0 & -\frac{1-d}{C} & 0 & -\omega & -\frac{1}{R C}
\end{array}\right]\left[\begin{array}{l}
x_{1} \\
x_{2} \\
x_{3} \\
x_{4} \\
x_{5} \\
x_{6}
\end{array}\right]+\left[\begin{array}{c}
\frac{d}{L} \\
0 \\
0 \\
0 \\
0 \\
0
\end{array}\right]\left\langle v_{i n}\right\rangle_{0}}
\end{aligned}
$$

\section{B. Closed-loop simulations}

To test behavior of arbitrary order GSSAM with closedloop control, buck converter is used as an example. In these simulations, duty cycle $d$ is determined by the control loop which receives inputs from the arbitrary order GSSAM model at deterministic rates, processes them and provides control signal update to the buck model. The control for buck converter consists of voltage and current regulators to determine the required duty cycle to achieve the set value. The set value for these simulations was $15 \mathrm{~V}$ with a step load change applied at $t=5 \mathrm{~ms}$ as shown in Fig. 7.

In addition to the closed-loop control, another feature of developed arbitrary order GSSAM has been explored. This is related to the ability to set different harmonic order for different state variables i.e. $k_{1} \neq k_{2} \neq \cdots \neq k_{q} \neq k$. The desired order can be set before the simulation. As an
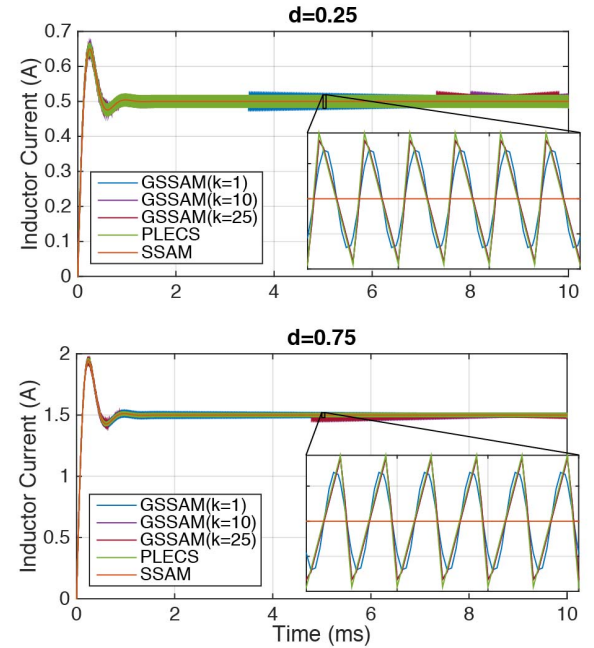

(a)
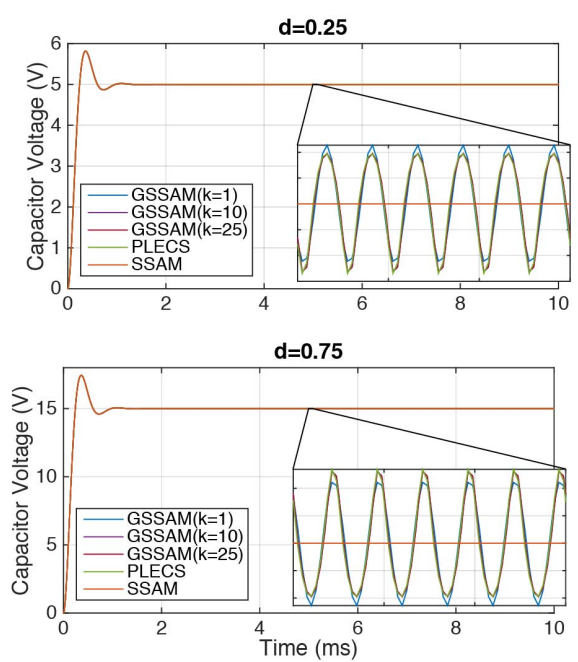

(b)

Fig. 4. Inductor current (a) and capacitor voltage (b) for different number of harmonics and duty cycles comparison with PLECS switched model for buck converter. 

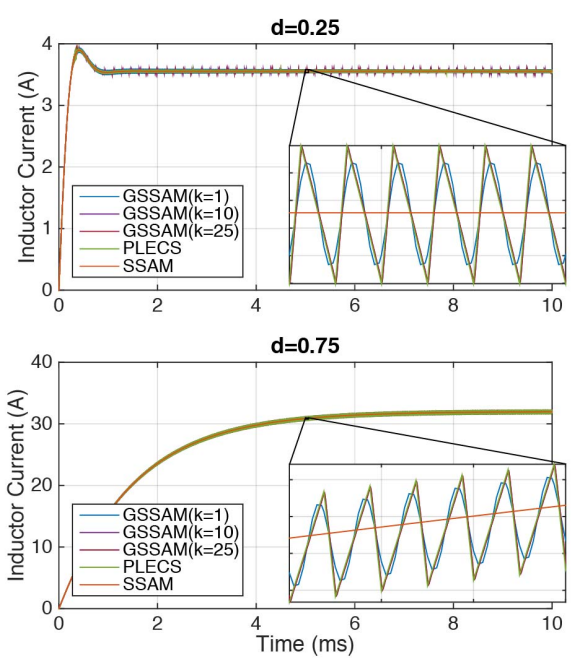

(a)
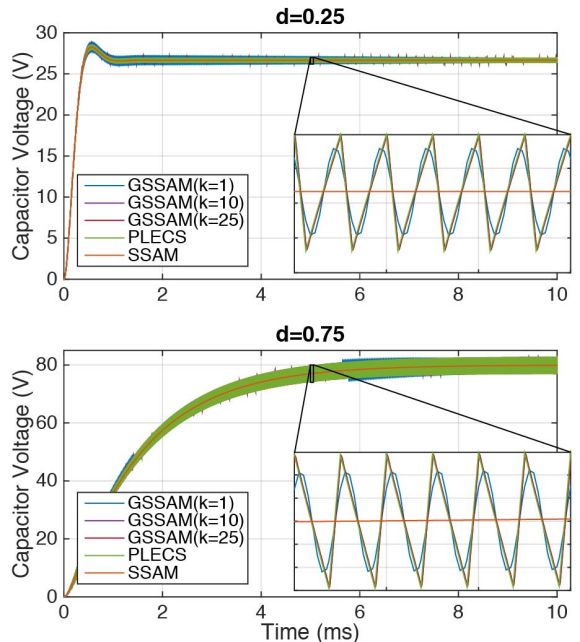

(b)

Fig. 5. Inductor current (a) and capacitor voltage (b) for different number of harmonics and duty cycles comparison with PLECS switched model for boost converter.
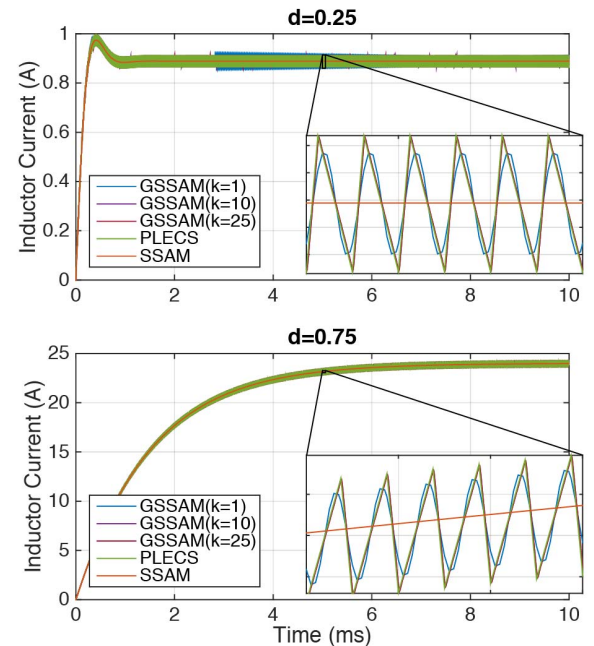

(a)
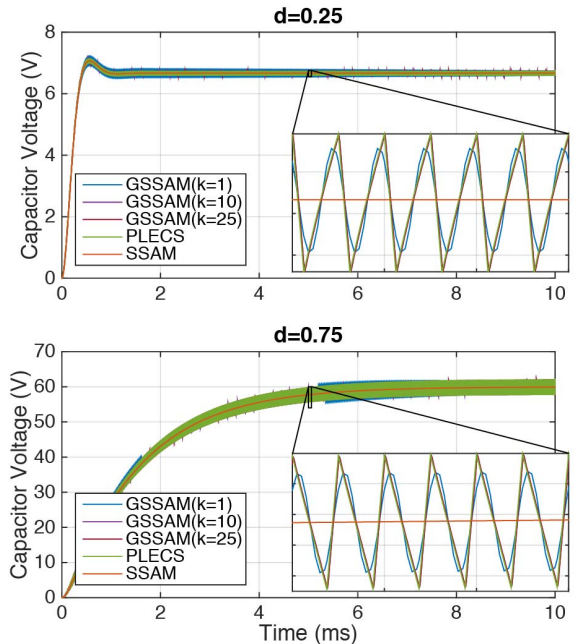

(b)

Fig. 6. Inductor current (a) and capacitor voltage (b) for different number of harmonics and duty cycles comparison with PLECS switched model for buck-boost converter.

example, three different cases are considered $k_{I}=k_{V}=1$, $k_{I}=7, k_{V}=4$ and $k_{I}=13, k_{V}=9$, indicating order of harmonics for state variables, respectively. The results of these simulations are as shown in Fig. 7. From Fig. 7, we can observe that the arbitrary order GSSAM models, with different harmonic order selected for each state variable, perform well in a closed-loop operation. The variation of duty cycle, due to controller actions, does not affect the GSSAM model. Similarly to open-loop case, addition of more harmonics e.g. $k_{I}=13, k_{V}=9$ to the GSSAM model, increases the fidelity and comes closer to the PLECS simulations.

Yet, more in-depth analysis is required to properly determine sufficient or optimal harmonic levels, which is beyond the scope of this paper.

\section{CONCLUSION}

The GSSAM models reported in literature mostly include only the 1st order harmonics in addition to dc part. While this improves the model's response, it is still unable to completely capture the switching dynamics of the devices. In this paper, we have explored possibilities to generalize the method and generate the arbitrary order GSSAM models (in terms of harmonic components being considered for each state variable) using Matlab. The algorithm presented can generate a mathematical model of an arbitrary order, based on the input from the user and having SSAM (zero-order) as a starting point.

Verification of developed models is done by open-loop and closed-loop simulations on a basic dc-dc converter topologies. 

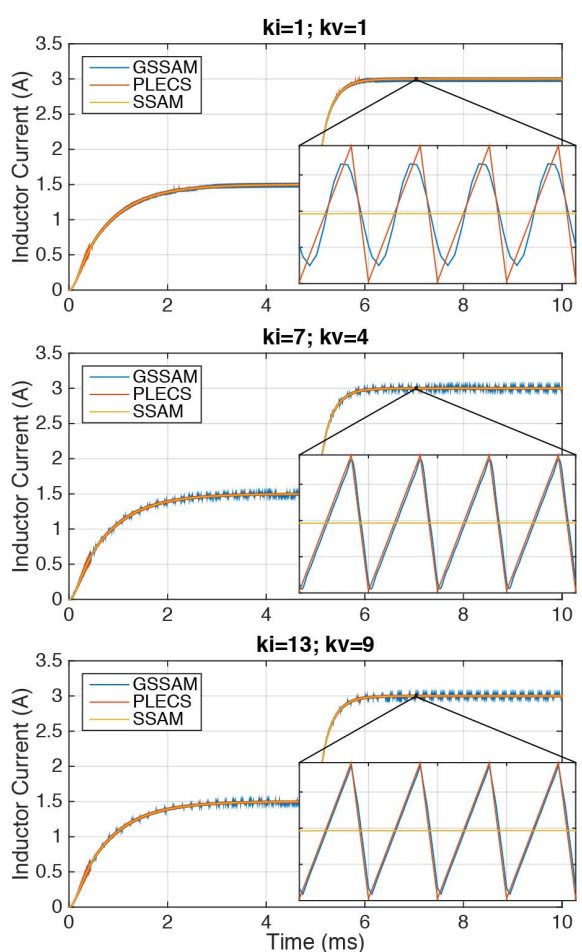

(a)
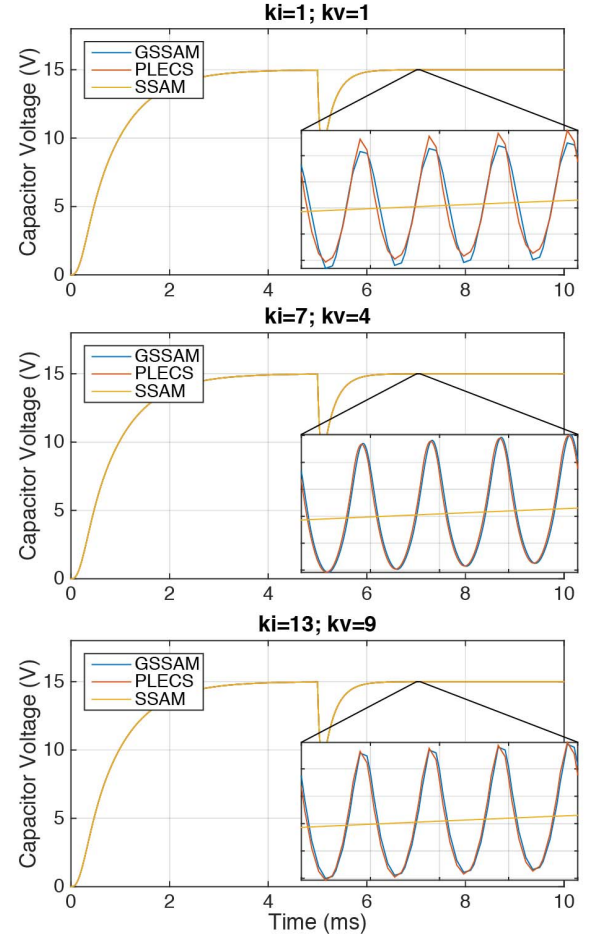

(b)

Fig. 7. Inductor current (a) and capacitor voltage (b) for closed-loop controlled Buck Converter and considering three different cases.

Increased number of harmonic components, brings simulation results closer to those of PLECS switched models. Order of harmonics used to represent the different state variables can be freely selected, as demonstrated in closed-loop simulations with buck converter, thus offering possibility to capture sensitive circuit behavior in the frequency range of interest.

\section{REFERENCES}

[1] J. Mahdavi, A. Emaadi, M. Bellar, and M. Ehsani, "Analysis of power electronic converters using the generalized state-space averaging approach," Circuits and Systems I: Fundamental Theory and Applications, IEEE Transactions on, vol. 44, no. 8, pp. 767-770, Aug. 1997.

[2] R. Middlebrook and S. Cuk, "A general unified approach to modelling switching-converter power stages," in Power Electronics Specialists Conference, 1976 IEEE, Jun. 1976, pp. 18-34.

[3] S. Sanders, J. Noworolski, X. Liu, and G. C. Verghese, "Generalized averaging method for power conversion circuits," Power Electronics, IEEE Transactions on, vol. 6, no. 2, pp. 251-259, Apr. 1991.

[4] V. Caliskan, G. C. Verghese, and A. Stankovic, "Multifrequency averaging of dc/dc converters," in Computers in Power Electronics, 1996., IEEE Workshop on, Aug. 1996, pp. 113-119.

[5] - "Multifrequency averaging of dc/dc converters," Power Electronics, IEEE Transactions on, vol. 14, no. 1, pp. 124-133, Jan. 1999.
[6] A. Emadi, "Modeling and analysis of multiconverter dc power electronic systems using the generalized statespace averaging method," Industrial Electronics, IEEE Transactions on, vol. 51, no. 3, pp. 661-668, Jun. 2004.

[7] Z. Lin and H. Ma, "Modeling and analysis of threephase inverter based on generalized state space averaging method," in Industrial Electronics Society, IECON 2013 - 39th Annual Conference of the IEEE, Nov. 2013, pp. 1007-1012.

[8] B. Zahedi and L. Norum, "Modeling, analysis and control of an isolated boost converter for system level studies," in Electrical Machines and Power Electronics and 2011 Electromotion Joint Conference (ACEMP), 2011 International Aegean Conference on, Sep. 2011, pp. 180-184.

[9] K. Corzine, X. Kou, and J. Baker, "Dynamic averagevalue modeling of a four-level drive system," Power Electronics, IEEE Transactions on, vol. 18, no. 2, pp. 619-627, Mar. 2003.

[10] A. Yazdani and R. Iravani, "A generalized state-space averaged model of the three-level npc converter for systematic dc-voltage-balancer and current-controller design," Power Delivery, IEEE Transactions on, vol. 20, no. 2, pp. 1105-1114, Apr. 2005.

[11] S. Lang, Undergraduate Analysis, 2nd ed. SpringerVerlag New York, 1997. 